\title{
Million per Gram
}

National Cancer Institute

\section{Source}

National Cancer Institute. Million per Gram. NCI Thesaurus. Code C98758.

A unit of measurement equal to one million entities per unit of mass equal to one gram. 